\title{
Self-Learning and optimization of processing volume in Data Centers under AKS Catalog Framework: A Survey
}

\author{
Aasheesh Raizada ${ }^{1}$ Kishan Pal Singh ${ }^{2}$ \\ ${ }^{1}$ Department of Computer Application, IET, Mangalayatan University, Aligarh, India. \\ ${ }^{2}$ Department of Mechanical Engineering, IET, Mangalayatan University, Aligarh, India.
}

\begin{abstract}
IT workload distributions are affecting data centers un-suitable airflow and temperature within the distribution. Traditional Computational Fluid Dynamics (CFD) is time-consuming and expensive, computationally and financially while the Reduced Order Models (ROM's) are fast as comparing towards. In AKS Extensible Catalog Framework (IAECF) contracting to ROM can do they without a user tolerance, and can make low errors using IAECF demonstrate for optimal workload distribution framework for processing in data centers. The optimization workload determination to lead approximately $8.2 \%$ (a year before comparing $2.4 \%$ growth) compared to energy savings with a particular uniform distribution with working on the basis of FFD improvement effective by the speed and multiplied-iteration for optimizing on data center eventually.The entire optimization process, including IAECF training in 3.2 hours in the design phase. This is an alternative methodology of CFD (based on Fast Fluid Dynamics) and preliminary study IAECF-based on optimizing algorithm placement platforms that are parallelizable and simple to program.
\end{abstract}

Key Words: Workload Optimization, Data Center, Online Learning, ROM Modules, Extensible Catalog Framework.

\section{INTRODUCTION:}

IT equipment that is effectively dissipated during operation as all input power heated in data centers during operations, as a heat removal method air cooling to frame the airflow from the cooling equipment towards the racks. A model has been employed for the airflow CFD widely comes for temperatures in data centers [1][2][3]. It leads to low down cooling efficiency (Tang et al. 2008). IT equipment approaches for providing cooling to the data center as used for optimizing the workload energy efficiency distribution that CRACs operate during lower airflow (Banerjee et al. 2010) while cooling down whitespace of the data center. Several algorithm workload placements proposed to maximize air temperature supply while ensuring server temperature explained by Moore et al. (2005). Tang et al. (2007) investigated on minimizing strategies on heat recirculation load placement. CFD has been employed for optimizing designs and airflow control, CFD simulations obtaining thermal map and with regard distribution on workload for data centers requiring computational efforts covering huge counting of scenarios. FFD is a fractional method consistently made for model advection taken in varies some researchers have come through the same method of Lagrangian [8] and several interpolation accuracy models reported [9]. FFD is an easy way for transient simulations, FFD has shown 30-1000 speed up (additional factor) on GPU [12][13]. The speed improved by FFD has shown that it can be a good tool for optimizing design and controlling applications. Therefore, FFD has a mixed accuracy. FFD is an intervening approach under CFD traditional or in nodal models, with low accuracy of FFD than CFD models traditional. For reducing the cost simplified in airflow models to get a fast prediction, zhang et al. (2009) explored a model under various airflow supplies in rack-inlet and in rack-load. An intimate assumption is considered as a virtual rack and a cooling unit, it is a combined medal for capturing local temperature arrangement or airflow pattern may fail. This paper is presenting a platform based on IAECF training for employing an FFD model that is running faster on GPU simulating the airflow in different workload supplies. CRAC supply increases temperature approximately $0.9^{\circ} \mathrm{C}$, this had $8.9 \%$ energy savings. If we compare the $\mathrm{K}-\mathrm{E}$ model from a algebraic simple model, both models give a comparable accuracy level, we found the reference platform in a data center with fairly airflow patterns. Although new research discovered to predict FFD with first-order finite upwind volume additional scheme, which proved essential with equal accuracy for simply based CFD and some simple applications like room ventilation and cooling temperature to the electronics. It shows a small difference between an algebraic and the $\mathrm{K}-$ E model in the data center whitespace considering enough the difference on electronic appliances with a faster recirculation so, the K-E model should be favoured towards at least, the applications plenum. For reducing the calculating time, researchers prepared an FFD presentation as an intervening method between CFD along with a multizone network of airflow models (zuo and chen 2009; zuo 2010; zuo et al. 2010). 


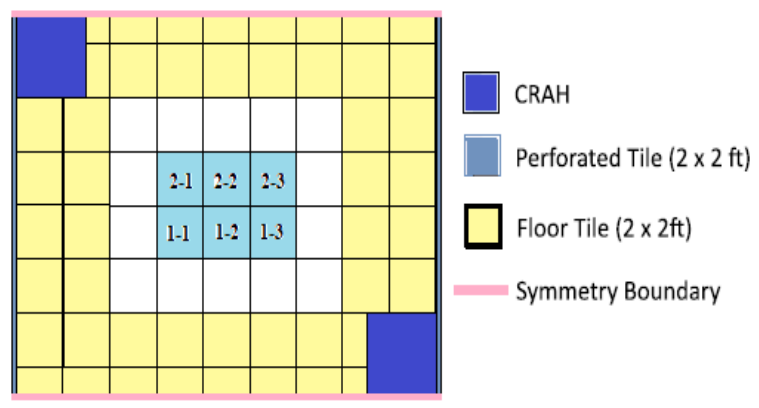

Figure 1.: Hypothetical Airflow Distribution Layout

CRAHs in data center distributes cooling air by air handles through tiles for IT inlet airflow can explore by designer and the operator. An FNM model has proposed by king et al. 2000 for predicting airflow rates. The assumption pressurized plenum can be applied on ideal scenarios for restrictive tile or in deep plenums. PFMs compute the airflow in the plenum everywhere using Navier-Stokes with balanced equations which CFD does. Using mathematical fractions in FFD can improve the speed by 32-39 times compared to CFD (Zuo and Chen 2009; Zuo 2010; Zuo et. Al-2010). Although CFD may be time-consuming to generate data, the ROM's almost instantaneously either interpolating using the data set. While traditional CFD are computationally expensive, used an iterative design on simulation cells. It can be seen if we train ROM with domain the system to operate the FFD model is a scale of simulation airflow at high speed. IAECF is a fast accelerating indoor simulation airflow using FFD, in we get computational grid and discretization specified order in FFD both CFD also using K-E model for solving significant faster. So, this study shows that the performance of the prepared model IAECF-FFD.

NOMENCLATURE TABLE:

\begin{tabular}{|l|l|}
\hline \multirow{2}{*}{ Symbols } & \multicolumn{1}{c|}{ Meaning and Definition } \\
\hline $\mathrm{K}$ & The velocity angle or vector \\
\hline $\mathrm{d}$ & The time and duration \\
\hline $\mathrm{X}$ & The structural coordinate \\
\hline $\mathrm{q}$ & The kinetic texture or viscosity \\
\hline $\mathrm{k}_{2}$ & The air density \\
\hline $\mathrm{t}$ & Temperature \\
\hline $\mathrm{P}$ & Pressure \\
\hline $\mathrm{f}$ & The source term \& interval source duration \\
\hline$\partial$ & (Jacobi's delta) Partial differential Sign \\
\hline$\Delta$ & (Delta) Change or difference in equation \\
\hline$\Delta \mathrm{P}$ & The Pressure drop across the tile \\
\hline $\mathrm{V}_{\mathrm{y}}$ & The velocity approaching the title \\
\hline$\beta$ & $\begin{array}{l}\text { (Beta)- It is used to denote a number of } \\
\text { variables with specific meaning }\end{array}$ \\
\hline $\mathrm{X}_{\mathrm{i}} \& \mathrm{X}_{\mathrm{j}}$ & Structural Coordinates \\
\hline $\mathrm{f}_{\mathrm{i}}$ & Force \\
\hline $\mathrm{S}_{\mathrm{t}}$ & Source \\
\hline $\mathrm{Y}^{+}$ & Structural Coordinates in wall unit \\
\hline
\end{tabular}

\section{METHODOLOGY:}

The Working of IAECF and FFD for Optimization in Data Centers for Workload:

The study of IAECF has dynamically established the lookup table, which is based on a present query that can be evaluated quickly by the linear local regressions (Pope 1997). In every training with evaluation stages, it answers the latest query, IAECF determines the nearest record of the IAECF table and estimates the outputs by using a linear approximation within the point of query in Ellipsoid Accuracy (EA). If the point of the query is outside of (EA) in the nearest record. IAECF simultaneously preform the local linear approximation and also a direct evaluation calling by CFD simulations for determining the outputs. For accelerating the process of training, employs the running of FFD on graphics unit processing 


\section{International Advanced Research Journal in Science, Engineering and Technology}

Vol. 8, Issue 6, June 2021

DOI: $10.17148 /$ IARJSET.2021.86125

to performing an airflow thermal simulation. FFD is a faster alternative from the traditional CFD. FFD solves similar governing equations which also CFD does, but it employs another technique of solving queries. FFD reported 60 times faster than the CFD with an additional component of 30-1000 speed up the time which can be achieved by using a parallel computation (Zuo and Chen 2010; Tian et. Al. 2017).

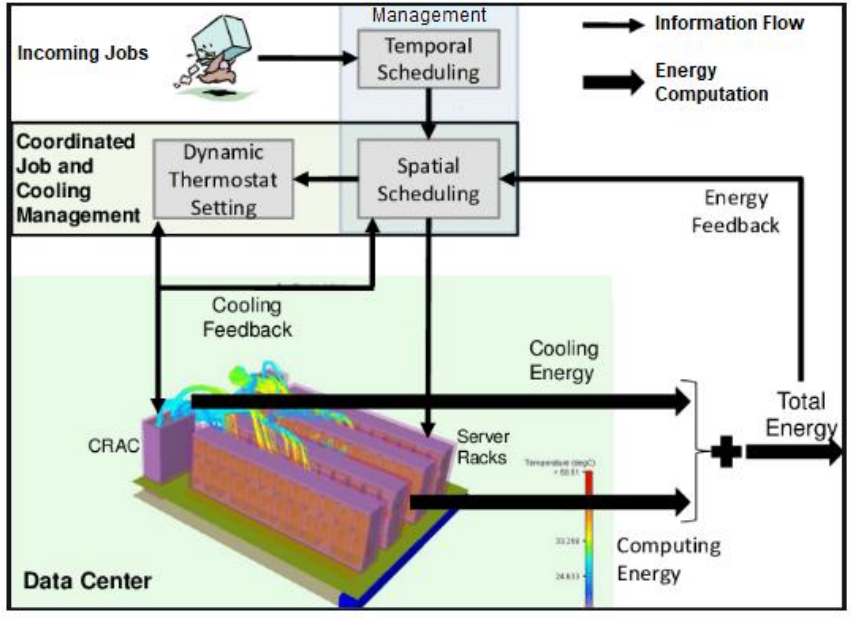

Figure.2: IAECF airflow during cooling and coordinated job management (Source: Research Gate)

\section{Optimization by Workload distribution IAECF and FFD:}

In this paper, the study of optimization performed the IAECF fully offline training by using the simulations of the (FFD). Figure1, show the platform optimization in the links of IAECF-FFD by using genetic algorithm (Mitchell 1998) which developed for automatic processing of whole training evaluation in IAECF for the optimization in the genetic algorithm. The optimization of conventional airflow evaluates a fitness function (which also called the objective function, that conduct, foremost thermal comfort) directly performed by using airflow simulations in CFD of FFD for predicting the temperature distribution and also the indoor velocity and then the predicted mean vote is calculated which evaluates the thermal comforts. The conventional method is different from, the idea in the optimization platforms it first trains reduced in the older model of IAECF in the data by series that is extracted by the simulations of airflow.

\begin{tabular}{|l|l|}
\hline Traditional CFD Method & \multicolumn{1}{|c|}{ FFD Method } \\
\hline $\begin{array}{l}\text { Compressed systematic } \\
\text { airflow }\end{array}$ & $\begin{array}{l}\text { Compressed systematic } \\
\text { airflow }\end{array}$ \\
\hline $\begin{array}{l}\text { Zero-representation sum } \\
\text { calculation of turbulent } \\
\text { airflow model }\end{array}$ & $\begin{array}{l}\text { Zero-representation } \\
\text { calculation of turbulent } \\
\text { airflow model. }\end{array}$ \\
\hline $\begin{array}{l}\text { RNG k- } \varepsilon \text { model with } \\
\text { airflow turbulence }\end{array}$ & $\begin{array}{l}\mathrm{Vt}=100 \mathrm{v} \text { with airflow } \\
\text { turbulence }\end{array}$ \\
\hline
\end{tabular}

Table 1. Exceptional perspective technique proposal used for assessing the method of FFD

For example, we use a various data series including boundary conditions (by using inputs) and also contain average the corresponding velocity with the temperature at occupant zone (containing outputs) for training the IAECF for storing data in the database.

\section{Data Centers Prediction Reference in FFD and CFD by IAECF:}

By using a genetic algorithm it generates a candidate solutions series for the initial population, which are sorted and evaluated with predicted fitness function of IAECF. By selecting the mutation and crossover for better solutions will inherit for the next generation with high probability, the iterations above procedures of the genetic algorithm are conversed as the optimal solution which is exported. By evaluating the performance of the solutions of the candidate by using the retrieve (known as linear regression) for various cases which have the fastest airflow simulations. 


\section{International Advanced Research Journal in Science, Engineering and Technology}

Vol. 8, Issue 6, June 2021

DOI: $10.17148 / I A R J S E T .2021 .86125$

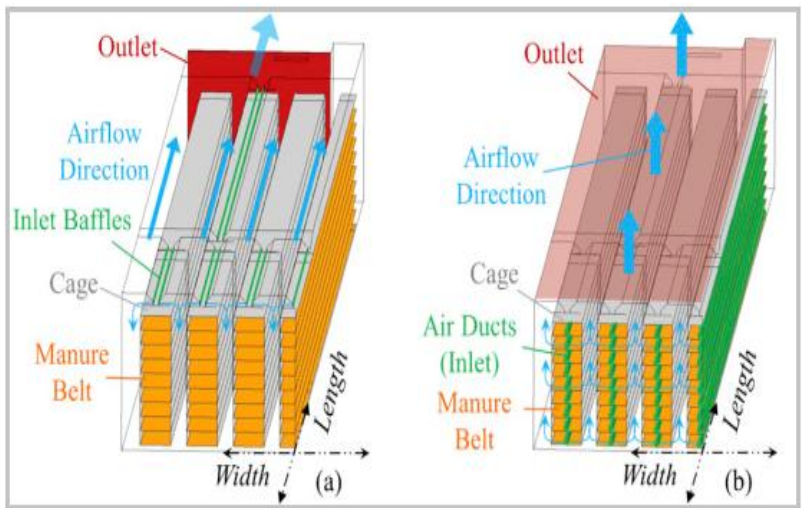

Figure 3.: Developed Simulation of CFD airflow ventilation displacement system (Source: Science Direct.com)

All the racks of IT have a capacity of the $54 \mathrm{U}$ in 18 racks which are located by the system of the distribution ( 1 row and 10 networking racks of $54 \mathrm{U}$ ), the measurements include temperatures of the racks which are used in the current study. The difference between the remaining an ultimate point which has Comparision made the methods by employing be FFD and CFD for the coupling of the velocity-pressure. The implementation of the FFD utilized finite volume for upwind discretization by the momentum and equations of the energy for an identical reference utilized CFD - traditional code.

\section{ANALYSIS OF ENERGY - REDUCTION PROSPECTIVE:}

The CRAC supplied the temperature of the air that can be scaled by the inlet rack temperature which reached the maximum recommended threshold for improving the efficiency of cooling by ASHRAE. In the current case, the rack inlet maximum temperature is decreased to $0.7^{\circ} \mathrm{C}$ by the benchmark for optimizing the workload of data in data centers, the air temperature supply is done by CRACs which can raise from $20.6^{\circ} \mathrm{C}$ to $21.8^{\circ} \mathrm{C}$. It is totally based on an empirical formula described in (Moore et. al. 2005), the performance of coefficient (POC) is valued for the increased form which is 3.42 to 3.66 , the result of the energy-saving is contained $1.1 \mathrm{~kW}$. The analysis of the energy reduction is estimated as $8.9 \%$ by workload distribution optimization.

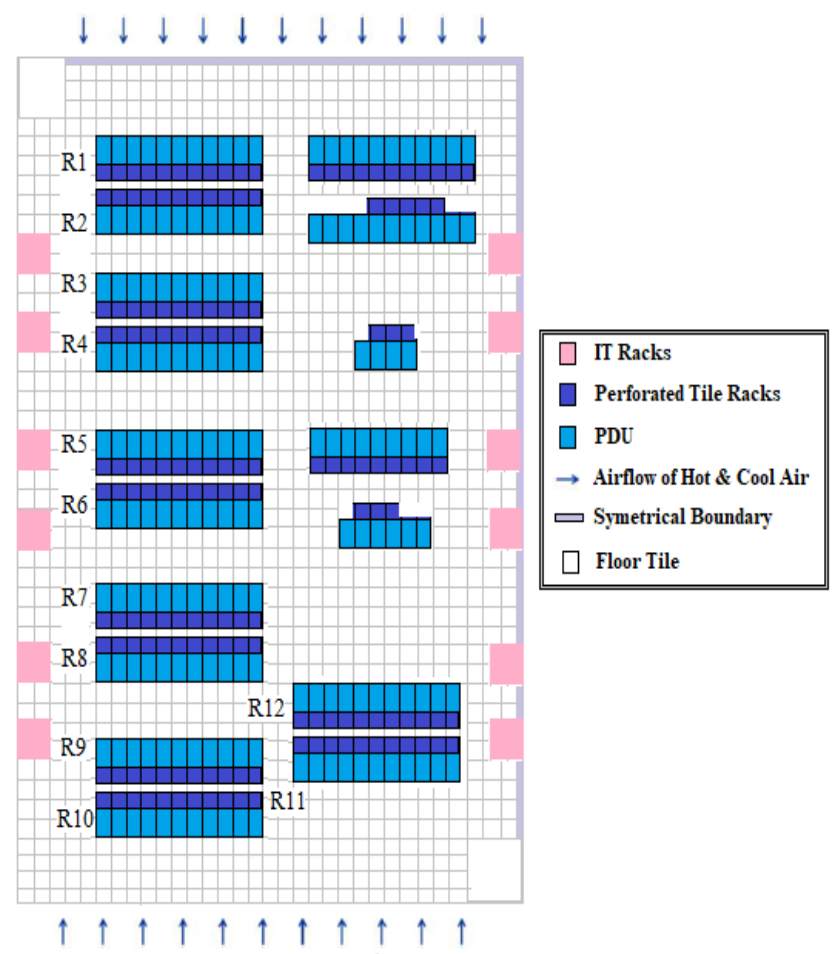

Figure 4.: Graphic Reference of the Data Center 


\section{International Advanced Research Journal in Science, Engineering and Technology}

Vol. 8, Issue 6, June 2021

DOI: $10.17148 /$ IARJSET.2021.86125

The IAECF table shown an accuracy using 50 data points randomly for each case, the prediction showing accuracy through the user total tolerance under training. Usually, the IAECF total error specified tolerance in the training stage for achieving a balance between training cost and prediction accuracy, which proper error tolerance may contain $0.7^{\circ} \mathrm{C}$ in this specialised case.

The working method is dived into two parts:

1. The optimization of workload differentiates between FFD and CFD prediction.

2. The adaptive tabulation learning model will context the data centers references and also compare the faculty located in data centers. In other part of the simulation is compared with the experimental data gathered by data centers, measurements include the rack-inlet temperatures for the current study.

\section{CASE-STUDY:}

The management area of the data airflow the center contains the temperature inlet-rack by the critical parameter for evaluating the performance of cooling, which can be influenced by various factors of the management such as air temperature is supplied by CRAC and the racks which have airflow patterns (which can lead to making hot and cool air by mixing completely).

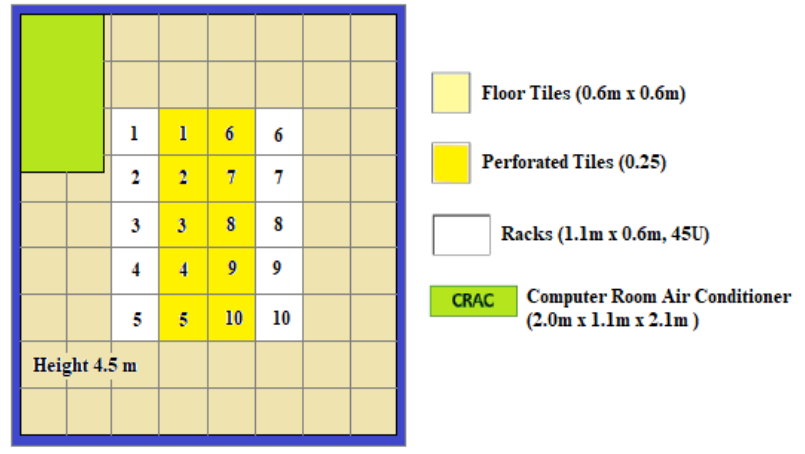

Figure 5.: Hypothetical Interpretation of the elementary Data Center

In a conclusion, the maximum temperature of the rack-the inlet is increased due to the hot local spots that are caused by a hot air mixture with the cold air in the front of the rack in the system of the cooling has a low supply in the temperature of the air and also increased the supply of flow rate, which conducts the lower efficiency to the system of cooling. The distribution of workload, in this paper focusing in the significant effect on the pattern of airflow nearby the racks and other influences in the temperature of the rack inlet that raised because of the hot local spots occurred by the mixing of the old and hot air front of racks the system of cooling and optimizing eliminate the supply by increasing the workload distribution and also improving the temperature of the cooling.

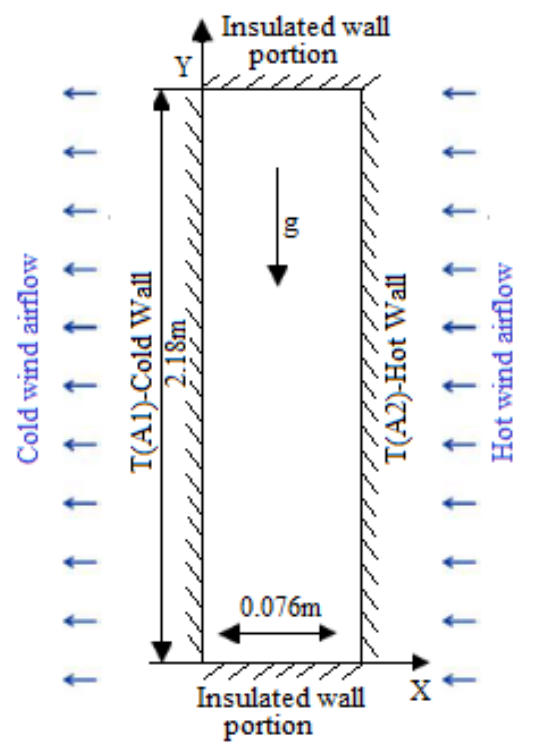

Figure 6.: Temperature Conditions in Inlet-rack airflow 
DOI: $10.17148 / I A R J S E T .2021 .86125$

\section{Comparing Turbulence Models:}

Now, comparing turbulence models to modelling whitespace - both one another compared to experimental measurements.

1. First, we add a momentum random source another to grid cell far more accurate "jet effects" perforated able on airflow.

2. The PDUs to the heat fluxes surface, were previously modelled as they were scale adiabatic.

So, compare as in a practically turbulence models in no longer hourly - simplistic viscosity approximate model for data centers whitespace.

The K.E. equations model is typical in the default choice for other applications Dhoot et. al. [18] prepared an algebraic model that makes an approximating wall function in the Chen to Xuo model improve accurately for electronics applications.

Discovering algebraic models required less calculation per iteration and it too simple than the K.E. model declaring faster overall, which also affects the scale of convergence rate. The center of fluid-cell adds computational cost.

\section{Distributing Workload in Data Centers by Optimization:}

Workload distribution is based on IAECF rack using optimization in the racks. Although we get $0.6^{\circ} \mathrm{C}$ error tolerance including optimization results based on IAECF training. So, we set the population size including the number of generations maximum 100 with probabilities settle over within Crossover and also Mutation. The function of the fitness in optimization study for minimizing the maximum temperature of racks which was supplied flow rate of air by CRAC. The result is shown by optimization in table 2. Generally, contain three optimal cases for similar solutions. The value of process 1 and process 2 for checking the rate of range about $1.0-1.3 \mathrm{~kW}$ and $9.6-9.9 \mathrm{~kW}$, As a result, the maximum temperature of racks with distribution optimal workload is $15.6^{\circ} \mathrm{C}$ by the predictions of IAECF for three cases. By evaluating the IAECF optimization accuracy based on IAECF performed a workload optimization based on the FFD. These results show only a $0.1^{\circ} \mathrm{C}$ temperature difference between the predictions of the IAECF and the FFD results are taken from the three cases simulations of datasets. Figure 4 compare time duration and costs which was performed by different cases of IAECF optimization. The time calls of FFD optimised on the performance of the simulation roughly estimated in every FFD simulation and calls during IAECF optimization in case 1-2-3 using IAECF model in FFD performing IAECF optimization by putting call to IAECF, taking 72.1 per cent only 7.9 per and 7.3 per time in FFD-GA. Airflow Repletion Dynamics:

In this module, we discuss the feature of common modelling to the traditional CFD and also FFD by including with plenum governed administratively equations by the relevant conditions of the boundary. Repletion Dynamics focus on modelling in the raised plenum of the floor with common conditions of the boundary for representing airflow inlet-models and perforated tiles.

\section{Administrated Equations:}

Navier-Stokes described the momentum of simplified equations which are solved by the help of traditional FFD and CFD methods in data sets that are

$$
\frac{\partial \mathrm{K}_{\mathrm{i}}}{\partial \mathrm{d}}=-\mathrm{K}_{\mathrm{j}} \frac{\partial \mathrm{K}_{\mathrm{i}}}{\partial \mathrm{x}_{\mathrm{j}}}+\mathrm{q} \frac{\partial^{2} \mathrm{~K}_{\mathrm{i}}}{\partial \mathrm{x}_{\mathrm{j}} \partial \mathrm{x}_{\mathrm{j}}}-\frac{1}{\mathrm{k}_{2}} \frac{\partial \mathrm{P}}{\partial \mathrm{x}_{\mathrm{i}}}+\mathrm{f}_{\mathrm{i}}
$$

Where the velocity angle is defined as $\mathrm{K}$, the time and duration is $\mathrm{d}$, the structural coordinate is $\mathrm{x}$, $\mathrm{q}$ is for kinematic texture, the density of air represented as $\mathrm{k}_{2}$, pressure is $\mathrm{P}$ and interval source duration is known as $\mathrm{f}$.

\section{Relevant Conditions of Boundary:}

The walls of the plenum are modelled by using the "slip" and "non-slip" relevant boundaries for a symmetrical and physical wall of the boundaries as respectively. Every airflow of inlet-racks, are designed by CRACs or also classified and distributed overall consuming area in the racks. Tiles that are entered in the racks are represented for proceeding resistance:

$$
\Delta \mathrm{P}=\frac{1}{2} \mathrm{k}_{2} \mathrm{SV}_{\mathrm{y}}^{2}
$$

The drop pressure on the tile is $\Delta \mathrm{P}$, the approaching velocity in the tile is $\mathrm{Vy}$, and $\mathrm{s}$ stands for loss dimensionless coefficient, by estimating the manufactured data and an empirical method:

$$
S=\frac{1}{\beta^{2}}\left[1+0.5\left(x_{i}-\beta\right)^{0.75}+1.414\left(x_{i}-\beta\right)^{0.375}\right]--(3)
$$

Where in the perforated tile $\beta$ is the open zone proportion.

\section{Execution of FFD:}

The difference between the traditional CFD and FFD contain a primary technique, which is used for solving the governed administrative equations. The method of linked equations is a variant that is used by the traditional CFD, and the time- 


\section{International Advanced Research Journal in Science, Engineering and Technology}

Vol. 8, Issue 6, June 2021

DOI: $10.17148 /$ IARJSET.2021.86125

splitting method is used by FFD. In datasets there are various ways for splitting the valid equations, due to our FFD implementations the momentum of equations is break three portions:

$$
\begin{aligned}
& \frac{\partial \mathrm{k}_{\mathrm{i}}}{\partial \mathrm{d}}=-\mathrm{k}_{\mathrm{j}} \frac{\partial \mathrm{k}_{\mathrm{i}}}{\partial \mathrm{x}_{\mathrm{i}}} \\
& \frac{\partial \mathrm{k}_{\mathrm{i}}}{\partial \mathrm{d}}=-\mathrm{q} \frac{\partial^{2} \mathrm{k}_{\mathrm{i}}}{\partial \mathrm{x}_{\mathrm{j}} \partial \mathrm{x}_{\mathrm{j}}}+\mathrm{f}_{\mathrm{i}} \\
& \frac{\partial \mathrm{k}_{\mathrm{i}}}{\partial \mathrm{d}}=-\frac{1}{\mathrm{k}_{2}} \frac{\partial \mathrm{P}}{\partial \mathrm{x}_{\mathrm{i}}}
\end{aligned}
$$

Equation (4) has been solved by Semi-Lagrangian or upwind scheme of the first order. In the latter method, it ensures to be rigorous mass or in energy construction unliked in the Lagrangian-Semi scheme. Equation(s), namely similar in solving first-order of traditional finite volume. At last, equation (6) shows the continuity solved equation done together.

$$
\frac{\partial \mathrm{k}_{\mathrm{i}}}{\partial \mathrm{d}_{\mathrm{i}}}=0
$$

Used a method of projection-corrective, for more defined description accuracy of FFD. Therefore, the first scheme for upwind flow increases solving time in a small percentage compare to the semi-Lagrangian scheme, it can improve also by utilizing in a high order accurate scheme and also increase computation time.

$$
\begin{aligned}
& \frac{\partial}{\partial d} k_{i}=-K_{j} \frac{\partial}{\partial x_{j}}+q \frac{\partial^{2}}{\partial x_{j}^{2}} k_{i}+\frac{\partial}{\partial x_{j}} P+\frac{f_{i}}{S t} \\
& K_{i}=\frac{\partial x_{i}}{\partial d}+\frac{f_{i}}{\partial x_{j}}+\partial y^{+}
\end{aligned}
$$

Several approaches that ensure the airflow in computed field and rates of tiles with governed equations of Navier-stokes [2]. Such as, the performed tiles of the rack inlet get modelled directly as a term in momentum equations applied for adjusting the airflow in tiles drops in a solved manner which converges the tile airflow with rates. The technique of pressure correction is proposed from Van-Glider et.al in (2011), satisfy plenum pressure [6 and 7] in constant values until the coverage in flow field by equation 1 under all tiles are perforated and noted that FFD solver is paralysed to run GPU in open [L. Consistent by Tian et. Al. (2017)].

\section{Evaluation of FFD Implementation Accuracy}

The major purpose of the paper is to assess the accuracy of FFD implementation in plenum applications of data centers, which predictions are made by the airflow of FFD and CFD by using the system of turbulence model, distribution of grids and order of discretisation. It considers an actual simulation of data centers which are measured by the experiment of airflow tile rates. This model of the plenum of flow considered this study as a better practice of the simultaneous plenum model which is utilized by an open large area of tiles with raised floor for improving accuracy in data sets implementation and evaluation. The hypothetical example has a large feature of an open area in tiles (58\%) and this makes the relative difference between FFD and CFD. Data Centers real applications are dominated by large open tiles area $-25 \%$, which is justified by the simulation of the plenum in this case.

$$
\partial \mathrm{k}_{\mathrm{i}}=-\mathrm{K}+\frac{\Delta \mathrm{t}}{\mathrm{P}}+\mathrm{f}
$$

\section{Turbulence Model Impact Comparision between FFD and CFD:}

The academic case is given by (VanGilder and zhang, 2015). As given in figure 1, the data center; "Symmetric slice" contain $14 \mathrm{ft}(4.3 \mathrm{~m})$ by $18 \mathrm{ft}(5.5 \mathrm{~m})$ with a depth of the plenum of $1 \mathrm{ft}(0.3 \mathrm{~m})$. CRAHS with two identities located diagonally opposite with one another, delivering $3000 \mathrm{cfm}(1.6 \mathrm{~m} \mathrm{3} / \mathrm{s})$ airflow. The interflow in each CRAH angle 45 degrees horizontal and parallel to perforated tiles in the rows. For creating a higher airflow recirculation leading interest in every tile variation flowing air. Having 6 open areas by (59\%) perforated tile arrange in two rows shown here. 


\section{International Advanced Research Journal in Science, Engineering and Technology}

Vol. 8, Issue 6, June 2021

DOI: $10.17148 / I A R J S E T .2021 .86125$

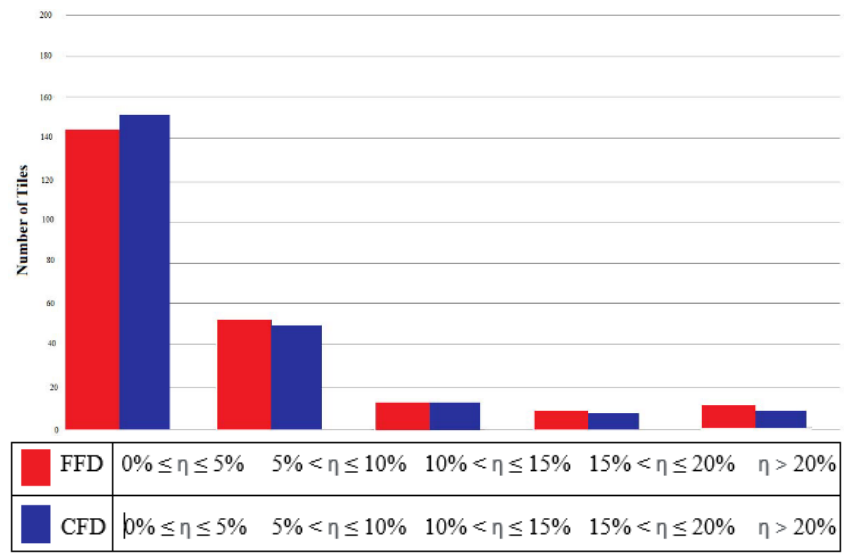

Figure 7.: Prediction of Inlet airflow of tiles categories by relative variation in majored experimental data

Facilitating comparison in several parameters of both FFD and also CFD comparing in such as numerical order, computational grid and also model of turbulence FFD directions in 6 uniforms $(0.15 \mathrm{~m})$ racks and in virtual direction there are 2 uniforms by $(0.05 \mathrm{~m})$ racks that give a grid system in $28 \times 36$ by framing 6 cells. FFD implementation has not had the KE-model option used in a 0 equation by which the viscosity fixed 100 times at molecular viscosity. This can't be best practice for modelling plenum in general, the accuracy here is not a primary goal. In the first discretization, the model was taken in both utilising the upwind scheme of FFD and CFD where appropriate. The FFD model is solved in $100 \mathrm{sec}$ in order of conservatively reaching. And on the other side, CFD simulates the inherent state. We show the rates of airflow by which perforated tiles in result shown in table 1 with FFD predictions allowing with CFD. There are relative maximum differences between airflow tile predictions by $3.6 \%$.

$$
\eta=\frac{\mid \text { Ysim }- \text { Ymea } \mid}{\text { Ymea }}
$$

Showing tile airflow obtain by the traditional CFD in popular turbulences of the K-E model utilizing significantly affect by turbulence model in tile airflow.

\section{CONCLUSION:}

This paper shows an optimization framework integrating IAECF on global optimization lead to seek distribution workload IT optimal. This platform demonstrates using FFD trained by IAECF and also used a hypothetical simulation data center with error tolerance $0.5^{\circ} \mathrm{C}$ of cost in IAECF training approximately 3.4 hours, in optimization, it takes 0.50 min additional. Comparing on the benchmark has a suitable workload distribution increasing the airflow temperature by $0.9^{\circ} \mathrm{C}$ save $70 \%$ cooling energy. This method is very helpful for reducing the cost of time and performing more and more cases using real layouts of data centers.

As FFD utilizes a fractional method that is shown so faster than CFD traditional, which utilities pressure velocity in simple based traditional method coupling. FFD is using design optimization with data center controlling applications often requiring multiplication simulations. FFD produced results solving indistinguishable of traditional CFD within data center plenum hypothetical application. First-order volume finite advection scheme ensuring grid into turbulence model implement consistently with both equations. This paper modelling the airflow and also the temperature in whitespace showing the data centre. Simulating FFD and CFD references from one to another. A turbulence modelling investigates plenums. FFD is an upwind first order volume employed in CFD. It can provide the same accuracy CFD in applications for the data centers. Shown fast to quantify but report robust more the CFD traditional convergence.

\section{REFERENCES:}

1. ASHRAE. 2015. Thermal Guidelines for Data Processing Environments. Fourth Edition ed.

2. Banerjee, A., T. Mukherjee, G. Varsamopoulos, and S. K. Gupta. 2010. Cooling-Aware and Thermal-Aware Workload Placement for Green Hpc Data Centers. Proceedings of the Green Computing Conference, 2010 International.

3. Demetriou, D. W., and H. E. Khalifa. 2013. Thermally Aware, Energy-Based Load Placement in Open-Aisle, Air-Cooled Data Centers. Journal of Electronic Packaging, 135 (3):030906.

4. Dhoot, P., J. W. VanGilder, Z. Pardey, and C. M. Healey. 2016. "Zero-Equation Turbulence Models for Large Electrical and Electronics Enclosure Applications." In.: Schneider Eletric.

5. Moore, J. D., J. S. Chase, P. Ranganathan, and R. K. Sharma. 2005. Making Scheduling" Cool": Temperature-Aware Workload Placement in Data Centers. Proceedings of the USENIX annual technical conference, General Track.

6. Tang, Q., S. K. Gupta, and G. Varsamopoulos. 2007. Thermal-Aware Task Scheduling for Data Centers through Minimizing Heat Recirculation. Proceedings of the Cluster Computing, 2007 IEEE International Conference on. 


\section{International Advanced Research Journal in Science, Engineering and Technology}

Vol. 8, Issue 6, June 2021

DOI: $10.17148 /$ IARJSET.2021.86125

7. Tian, W., T. A. Sevilla, D. Li, W. Zuo, and M. Wetter. 2018. Fast and Self-Learning Indoor Airflow Simulation Based on in Situ Adaptive Tabulation. Journal of Building Performance Simulation, 11 (1):99-112.

8. Tian, W., T. A. Sevilla, and W. Zuo. 2017. A Systematic Evaluation of Accelerating Indoor Airflow Simulations Using Cross-Platform Parallel Computing. Journal of Building Performance Simulation, 10 (3):243-55.

9. Tian, W., J. W. VanGilder, M. Condor, X. Han, and W. Zuo. 2019. "An Accurate Fast Fluid Dynamics Model for Data Center Applications." In IEEE ITherm Conference. Las Vegas, NV.

10. Zuo, W., and Q. Chen. 2009. Real-Time or Faster-Than-Real-Time Simulation of Airflow in Buildings. Indoor air, 19 1:33-44.

11. Zuo, W., and Q. Chen. 2010. Fast and Informative Flow Simulations in a Building by Using Fast Fluid Dynamics Model on Graphics Processing Unit. Building and Environment, 45 (3):747-57.

12. Jin, M., Zuo, W., Chen, Q., 2012. Improvements of fast fluid dynamics for simulating air flow in buildings. Numerical Heat Transfer, Part B: Fundamentals 62, 419-438.

13. Tian, W., Sevilla, T.A., Zuo, W., 2017. A systematic evaluation of accelerating indoor airflow simulations using cross-platform parallel computing. Journal of Building Performance Simulation 10, 243-255.

14. VanGilder, J.W., Pardey, Z.M., Bemis, P., Plamondon, D.W., 2016. Compact modeling of data center raised-floor-plenum stanchions: Pressure drop through sparse tube bundles, in: Thermal Thermomechanical henomena Electronic Systems (ITherm), $201615^{\text {th }}$ IEEE Intersociety Conference. IEEE, pp. 1148-1155.

15. VanGilder, J.W., Sheffer, Z.R., Zhang, X.S., Healey, C.M., 2011. Potential Flow Model for Predicting Perforated Tile Airflow in Data Centers. ASHRAE Transactions 117.

16. Zuo, W., Chen, Q., 2009. Real-time or faster-than-real-time simulation of airflow in buildings. Indoor air 19, $33-44$.

17. L. Marshall, and P. Bemis, "Using CFD for Data Center Design and Analysis", Applied Math Modeling White Paper, 2011.

18. J. Ferziger, and M. Peric, Computational Methods for Fluid Dynamics, Springer, 2002.

19. S. Patankar, Numerical heat transfer and fluid flow, CRC press, 1980.

20. W. Zuo, and Q. Chen, "Real-Time or Faster-than-Real-Time Simulation of Airflow in Buildings", Indoor air, vol. 19, no. 1, pp. 33-44, 2009.

21. M. Jin, W. Zuo, and Q. Chen, "Improvements of Fast Fluid Dynamics for Simulating Air Flow in Buildings", Numerical Heat Transfer, Part B: Fundamentals, vol. 62, no. 6, pp. 419-438, 2012.

22. W. Tian, T. Sevilla, and W. Zuo, "A Systematic Evaluation of Accelerating Indoor Airflow Simulations Using Cross-Platform Parallel Computing”, Journal of Building Performance Simulation, vol. 10, no. 3, pp. 243-255, 2017.

23. W. Abdelmaksoud, H. Khalifa, T. Dang, B. Elhadidi, R. Schmidt, and M. Iyengar, "Experimental and Computational Study of Perforated Floor Tile in Data Centers", Proceedings of Thermal and Thermo mechanical Phenomena in Electronic Systems (ITherm), Las Vegas, Nevada, June 2$5,2010$.

24. B. Launder, and D. Spalding, "The Numerical Computation of Turbulent Flows", Computer Methods in Applied Mechanics and Engineering, 1974. 